\title{
Percepción de empleadores sobre el Licenciado en Enfermería de la Facultad de Estudios Superiores Zaragoza
}

\section{Collection of employers on the degree of nursing school of graduate studies Zaragoza}

Mtra. Silvia Crespo Knopfler*, Mtra. María Susana González Velázquez*, Mtra. María Teresa Cuamatzi Peña* Lic. Leticia Domínguez Villanueva** y Lic. Lucía Ivonne González Nila**

*Profesoras de Tiempo Completo de la Carrera de Enfermería, FES Zaragoza UNAM

**Pasantes de la Licenciatura en Enfermería de la FES Zaragoza UNAM

\section{Resumen}

Introducción: Actualmente el mundo se enfrenta a una situación de mayor competencia comercial y económica entre los países, lo cual crea una necesidad de responder a las demandas que esta situación exige. Esta investigación nos permitió identificar la opinión de los empleadores sobre la formación y el desempeño profesional del egresado de la licenciatura en Enfermería. Objetivo: Determinar la percepción de los empleadores sobre el perfil del Licenciado en Enfermería e identificar en que aspectos es necesario reforzar su formación y facilitar su inserción laboral. Metodología: Se trata de un estudio descriptivo, transversal donde se utilizó un cuestionario dirigido a los empleadores del sector salud y el cual se calificó en base a la escala de Likert. Para la captura y elaboración de la base de datos se utilizó el programa estadístico SPSS versión 11.5. Resultados: El 43.5\% de los empleadores califican el desempeño laboral del licenciado como muy bueno;

Introduction: The world is currently facing a situation of greater trade and economic competition among countries, thus creating a need to respond to the demands that this situation requires. This research allowed us to identify the views of employers on training and performance of the graduate degree in nursing. Objectives: To determine the views of employers on the profile of Bachelor of Nursing and identify areas where there is a need to strengthen their training and facilitating their employability. Methodology: This is a descriptive study, and cross prolectivo where we used a questionnaire to employers in the health sector; scored based on the Likert scale. To capture and development of the database was used statistical program SPSS version 11.5. Results: $43.5 \%$ of employers describe the job
93\% de los empleadores consideran que es muy importante reforzar las habilidades en la toma de decisiones, $86 \%$ en técnicas y trabajo en equipo, $81.5 \%$ en conocimientos generales y proceso enfermero, $79 \%$ en conocimientos especializados, $76.8 \%$ en investigación y $58.2 \%$ en conocimientos básicos en ciencias biomédicas, sociales y de la conducta. Conclusiones: Los empleadores opinaron que el desempeño laboral del licenciado es muy bueno y están satisfechos con los conocimientos especializados, sin embargo, hacen énfasis en profundizar más en los mismos, así como reforzar la habilidad en la toma de decisiones, en técnicas, trabajo en equipo, conocimientos generales, aplicación del proceso enfermero y modelos y teorías de Enfermería.

Palabras clave: Empleadores, egresados, Enfermería.

\section{Abstract}

performance of licensed as very good, 93\% of employers consider it very important to strengthen skills in decision-making, $86 \%$ in techniques and teamwork, $81.5 \%$ in general knowledge process and nurses, $79 \%$ in expertise, $76.8 \%$ and $58.2 \%$ in research in basic knowledge in biomedical sciences, and social behavior. Conclusions: The employers felt that the degree of job performance is very good and are satisfied with the expertise, however, emphasize deeper into the same, as well as strengthen the ability in decision-making skills, teamwork, general knowledge, implementation of the process nurses and nursing theories and models.

Keywords: Employers, graduates, nursing. 


\section{INTRODUCCIÓN}

La educación superior en México, enfrenta un escenario de competencia mundial, lo que conlleva a la necesidad de plantear programas educativos que desarrollen una educación de calidad con pertinencia social y equidad y que respondan a las necesidades del sector laboral de las instituciones de salud. Es ahí donde adquiere una gran relevancia el perfil requerido de Enfermería para dichas instituciones.

La percepción del empleador acerca de la preparación que demuestra el licenciado en Enfermería con relación al perfil laboral deseado en el sector de salud público y privado en los diversos niveles de atención es de suma valía para las escuelas para contrastar éste, con el perfil profesional de egreso que plantea la escuela y establecer la coherencia entre el sector laboral y educativo.

El presente estudio tuvo como propósito determinar la opinión de los empleadores sobre el perfil del licenciado de Enfermería de la Facultad de Estudios Zaragoza con el fin de mejorar la formación profesional de los mismos y facilitar su inserción laboral en los diversos escenarios.

En función de que el empleador tiene una percepción acerca del perfil del egresado de la licenciatura en Enfermería sobre: conocimientos disciplinares, aplicación del marco ético legal de la profesión, sensibilidad para ofrecer una atención humanista, integración con el equipo multidisciplinario, disposición del trabajo en equipo, conocimiento del campo de desarrollo profesional, gestor del cuidado de Enfermería y habilidad para la toma de decisiones entre otros, lo cual brinda criterios para comparar el desempeño de los egresados de la carrera.

Además de estos elementos, actualmente los profesionistas egresados deben poseer competencias transferibles que les permitan desempeñarse en diferentes áreas ocupacionales y con un acervo de capacidades y habilidades vinculadas con el pensar; para prevenir y resolver problemas y toma de decisiones; flexibilidad mental, pensamiento reflexivo, sentido de anticipación; actitudes creativas y preactivas; formación básica general; capacidad de comunicarse, negociar, conciliar, saber escuchar; autoestima, búsqueda de desafíos y trabajo en equipo ${ }^{1,2}$.

En la actualidad la formación de un profesionista tiene tres aspectos básicos: a) el desarrollo del conocimiento general, b) el desarrollo del conocimiento laboral y c) la experiencia en el trabajo, señala Gonczi ${ }^{3}$, ha sido tarea difícil para las instituciones de educación superior integrar la teoría y la práctica en el contexto social, cultural y productivo, en el cual el futuro profesionista se desempeñara.
Según Coronado y Tapia 4 , la vinculación universidadsector productivo, surge de la relación educación-economía que se plantea como parte de los requerimientos educativos que están asociados a la globalización económica y a la apertura comercial. No obstante esta relación debe estar cimentada prioritariamente por la calidad de la atención que requiere nuestra población del personal de Enfermería y solo a través de la investigación evaluativo se puede establecer el grado en que se logran los perfiles de egreso de los alumnos que formamos y retroalimentar los programas de estudio y la formación de los docentes e infraestructura que se conjugan para ello, a través de las aportaciones de las instituciones de salud.

Es importante mencionar que se han realizado estudios previos en nuestro país como el de Salazar y cols. (2006) de la Facultad de Enfermería de la Benemérita Universidad Autónoma de Puebla en donde el personal directivo de Enfermería de las diferentes instituciones de salud califican el desempeño profesional de los egresados como bueno en diferentes rubros como son: conocimientos de la disciplina, integración a la misión institucional, a la aplicación de principios éticos, atención humanitaria integración multidisciplinario, entre otros.

\section{METODOLOGÍA}

Se realizó un estudio descriptivo, transversal de acuerdo a la clasificación de Hernández y cols. ${ }^{5}$ El cuestionario que se utilizó fue validado por expertos en el área y piloteado haciendo los ajustes pertinentes. Este instrumento estuvo constituido por ocho apartados que son: datos personales del empleador, requisitos formales para la contratación, desempeño profesional del licenciado (a), valores y actitudes, habilidades, aspectos importantes a reforzar para la formación del licenciado de Enfermería y una parte cualitativa constituida por las observaciones de los empleadores, Se utilizó una hoja de consentimiento informado para su aplicación. El instrumento se calificó en base a la escala de Likert con las siguientes opciones: (siempre, casi siempre, a veces casi nunca y nunca) y (muy importante, importante, poco importante, nada importante); el grado de confiabilidad fue de 0.60 al obtener el Alpha de Cronbach. Para el levantamiento de datos se utilizó la entrevista aplicada a 43 empleadores de 14 instituciones del Sector de Salud pública y privado, en el sector público se opto en los tres niveles de atención. La muestra fue no probabilística de conveniencia al investigador. El criterio de inclusión para la entrevista con los empleadores fue que tuvieran egresados de la Facultad 
a su cargo y que aceptaran contestar el cuestionario. Para la captura y elaboración de la base de datos fue utilizado el programa estadístico SPSS versión 11.5. En el análisis se empleó por cada variable las frecuencias y porcentajes de las mismas.

\section{RESULTADOS}

Para el proyecto se tomaron en cuenta 14 instituciones del Sector Salud, de las cuales el $57.2 \%$ pertenece a la Secretaría de Salud, el 14.3\% al Instituto Mexicano del Seguro Social (IMSS), 14.3\% al Sector Privado, 7.1\% al Instituto de Seguridad Social para Trabajadores del Estado (ISSSTE) y el $7.1 \%$ al Instituto Social del Estado de México (ISEM). Del total de instituciones entrevistadas, el $21.4 \%$ pertenece al primer nivel de atención, 35.5\% al segundo nivel y el $42.7 \%$ al tercer nivel. En relación al área laboral del empleador, el servicio de hospitalización obtuvo el $23 \%$ siendo el de mayor frecuencia. Con relación a la edad, se observó que el $30.2 \%$ de los empleadores se encuentran entre los 31 a 40 años de edad y con respecto al sexo es importante mencionar que el $93 \%$ de los empleadores es del sexo femenino.

Con respecto al puesto que ocupan los empleadores, se observó que de los 43 entrevistados, el $44.1 \%$ son jefes de servicio, $23 \%$ supervisores y el $32.9 \%$ restante son enfermeras generales, médicos, jefes de enfermeras, coordinadores de enseñanza y subjefe de educación médica. Cabe mencionar que el $74.4 \%$ de los empleadores tienen a su cargo de 1 a 10 licenciados de Enfermería de la FES Zaragoza.

Con relación al sector en donde laboran los egresados, el $85.5 \%$ se encuentra laborando en instituciones públicas y solo el $14.5 \%$ en instituciones del sector privado.

El requisito más importante para la contratación por parte de los empleadores, fue tener título profesional con un $67.5 \%$; en segundo orden de importancia fue la entrevista formal con un $37.7 \%$ y en tercer lugar los exámenes de selección 29\%.

De las características personales deseables para la contratación, los empleadores le conceden a la edad de los licenciados un $72 \%$ mayor de importancia que al sexo y al estado civil. Otro aspecto importante para la contratación son las características de personalidad en donde el $97.6 \%$ de los empleadores le concede la más alta importancia a la buena presentación, seguida del 93\% las habilidades para la toma de decisiones, el $79 \%$ en capacidad de liderazgo y el $51.1 \%$ en sentido del humor.

Respecto al rubro sobre conocimiento, el $90.7 \%$ de los empleadores consideraron de alta importancia el trabajo en equipo; seguida del $88.3 \%$ la habilidad para la comunicación oral y escrita y $72.1 \%$ en el manejo y búsqueda de información.

En lo que se refiere al desempeño profesional del licenciado, los empleadores manifestaron estar totalmente satisfechos con la habilidad en la toma de decisiones en un $44.9 \%$ y asumir responsabilidades con el $46.4 \%$, aunque es importante mencionar que son porcentajes bajos.

En los aspectos donde los empleadores están satisfechos son: la habilidad para la resolución de problemas con un $46.4 \%$ y en lo que respecta al conocimiento del marco ético-legal con el $60.9 \%$. El empleador se encuentra satisfecho con los conocimientos básicos en ciencias biomédicas, sociales y de la conducta en un $56.5 \%$, totalmente satisfecho con el $27.5 \%$ y poco satisfecho con el $13 \%$.

Al preguntar a los empleadores ¿cómo es el desempeño laboral del licenciado? respondieron que es excelente con un $24.6 \%$; muy bueno $43.5 \%$, bueno $27.5 \%$ y regular $4.3 \%$ (Cuadro 1).

Con relación al desempeño del licenciado en el rubro de valores y actitudes, el $79.7 \%$ de los egresados siempre ejercen con responsabilidad sus funciones y muestran respeto a los pacientes, el $78.3 \%$ siempre muestran respeto a sus superiores, y el $76.8 \%$ siempre muestran interés y disposición para realizar el trabajo.

Los empleadores consideraron que es muy importante reforzar en los egresados las habilidades para la toma de decisiones en un $93 \%$, técnicas y trabajo en equipo con $86 \%$, conocimientos generales y proceso enfermero con $81.5 \%$, conocimientos especializados con $79 \%$, investigación, gestión y administración con $76.8 \%$ y los conocimientos básicos en ciencias biomédicas, sociales y de conducta con 58.2\%, (Cuadro 2).

\section{DISCUSIÓN Y CONCLUSIONES}

Se observó que la mayoría de los egresados (85.5\%) se encuentran laborando en el sector público, donde coincide con los resultados obtenidos en la investigación de Sánchez ${ }^{6}$, donde los profesionales de la salud (81.5\%) están empleados en empresas públicas y con la de Salazar en donde se obtuvo que 63 egresados de las primeras cuatro generaciones se encuentran laborando en instituciones públicas y 49 en el régimen privado, dando un total de 112 egresados empleados.

En los datos obtenidos, los empleadores que se entrevistaron tienen el puesto de jefes de servicio, supervisores, jefes de enfermeras, jefe y coordinador de enseñanza, lo 
que se relaciona con Salazar ${ }^{8}$ en donde el personal que entrevistado fueron directivos de diferentes instituciones de salud del régimen público y privado, con los cargos de jefe de enfermeras, subjefe y coordinador de enseñanza.

Dentro de los requisitos formales para la contratación, en nuestro estudio se encontró que el título fue el más importante con un $67.5 \%$, seguido por los exámenes de selección con el $34.8 \%$, lo cual presenta similitud a los estudios de Sánchez ${ }^{9}$ y con el de Estrella y Ponce ${ }^{10}$ en donde poseer un título profesional influye mucho en la contratación, de igual manera, se confirma con el estudio de Salazar ${ }^{11}$ donde el requisito formal de aceptación fue tener título profesional y pasar una entrevista formal.

Entre los requisitos deseables para la contratación, los empleadores opinaron que el más importante dentro de la categoría de personalidad es la buena presentación con un 97.6\%, coincidiendo con el estudio de Estrella y Ponce ${ }^{12}$, donde se menciona que influye mucho para la contratación esta misma característica.

En otro rubro los empleadores están en un 39.1\% totalmente satisfechos con el desempeño profesional del licenciado para asumir responsabilidades y en conocimientos generales con un $34.8 \%$, coincidiendo con el estudio de Valencia $(2006)^{13}$, donde destacan en este rubro la disposición de aprender, el asumir responsabilidades y los conocimientos generales de la disciplina.

En relación al proceso enfermero dentro del desempeño profesional del licenciado, los datos mostraron que los empleadores están totalmente satisfechos con un 39.1\%, que aún siendo el de mayor porcentaje sigue siendo bajo, con un $39.1 \%$ satisfecho y $18.8 \%$ poco satisfecho, lo que coincide con el estudio de Gutiérrez (2004) ${ }^{14}$ donde la utilización del proceso enfermero como herramienta para el cuidado le otorgaron una calificación de satisfactorio en un $43 \%$.

Los empleadores en general, califican el desempeño laboral del licenciado como muy bueno (43.5\%) que aún siendo el más alto posee un porcentaje menor al 50\%, lo que coincide con los estudios de Gutiérrez ${ }^{15}$ y Valencia ${ }^{16}$.

En lo que respecta al apartado de valores y actitudes los más importantes fueron: ejercer con responsabilidad con un $79.7 \%$, el respeto a los pacientes; con $79.7 \%$, el respeto a los compañeros ;con $75.4 \%$; el respeto a sus superiores con un $78.3 \%$, interés y disposición para realizar el trabajo con el $76.8 \%$, la colaboración con sus compañeros en un $73.9 \%$ y la colaboración con sus superiores con un $75.4 \%$; coincidiendo de este modo con el estudio de Sánchez ${ }^{17}$, donde los valores y actitudes más señaladas fueron la disposición al trabajo con el 25.43\%, participación y colaboración con $14.6 \%$, la responsabilidad con $32.3 \%$ y el respeto con un $25.96 \%$, los cuales tienen porcentajes bajos aun siendo los que más se mencionaron, de la misma manera se relaciona con los hallazgos de Estrella y Ponce ${ }^{18}$ donde la responsabilidad es el valor que más reconoce el empleador con un $70.8 \%$.

Por último es importante mencionar que dentro de los aspectos más importantes a reforzar en la formación del licenciado, fueron los conocimientos especializados con el $79 \%$ y habilidad para la toma de decisiones con un $93 \%$, lo que es congruente a lo reportado por Estrella y Pon$\mathrm{ce}^{19}$, donde los temas de mayor interés en las necesidades de la formación son los disciplinares y especializados con el $40.6 \%$ y, de acuerdo con Salazar ${ }^{20}$, los egresados que laboran mencionaron que en las instituciones en que trabajan les exigen demasiado sobre conocimientos generales de la disciplina y en la habilidad para la toma de decisiones.

En relación a la parte cualitativa del instrumento, los empleadores de dos instituciones pertenecientes a la Secretaria de Salud mencionan, dentro de las observaciones, que los licenciados no aplican el proceso enfermero y no se basan en las teóricas de Enfermería, además de que les falta actuar más con humanismo y cortesía con los pacientes.

\section{CONCLUSIONES}

Al ser el primer acercamiento sobre la percepción de los empleadores en el plano laboral de los alumnos egresados de la licenciatura de la Facultad de Estudios Superiores Zaragoza, los hallazgos permiten vislumbrar fortalezas y debilidades que deben ser estudiadas detenidamente y realimentar el plan de estudios; las principales conclusiones versaron sobre:

- El mayor número de egresados se encuentra laborando en el tercer nivel de atención.

- En general, los empleadores, califican el desempeño laboral del egresado de la licenciatura en Enfermería de la FES Zaragoza, acorde a su puesto como muy bueno.

- La mayoría de los empleadores, refieren que los requisitos formales más importantes para la contratación son: título profesional, pasar una entrevista formal y acreditar los exámenes de selección.

- De las características deseables para la contratación, las más importantes para los empleadores son la edad, la buena presentación y el trabajo en equipo. 
- Los empleadores refieren estar satisfechos con los conocimientos especializados, sin embargo, hacen énfasis en que se debe profundizar más en los mismos.

- En percepción de los empleadores el Licenciado de Enfermería siempre ejerce con responsabilidad sus funciones, proporcionan respeto a los pacientes y a sus superiores, muestra interés, colaboran con sus compañeros y superiores y muestran disposición para realizar el trabajo, sin embargo el empleador menciona que se debería reforzar el trabajo en equipo, toma de decisiones, aplicación del proceso enfermero y profundizar en conocimientos de especialización, estos dos últimos requerimientos deben tener un espacio de reflexión sobre si la formación debe ser en las licenciadas de Enfermería como especialista, situación que requiere estudios de posgrados y sí el proceso enfermero se desarrolla cotidianamente en los servicios de salud.

\section{REFERENCIAS BIBLIOGRAFICAS}

1 Gutiérrez D. Educación para el trabajo. Revista Transición Educación, ¿Nuevo Rumbo?, Centro de Estudios para la Transición Democrática, Veracruz 1999: 1-3.

2 Vargas, L.M. La educación superior no responde a las demandas de los empleadores,UPAEP, 2003, (on line). (consultado 17/ Vl/ 2008) Disponible en http://universia.net.mx. México: 1-15, Encontrado en Acceso el 17 de junio de 2008

3 Valenzuela E. Ponce L.M. Impacto laboral de los egresados universitarios y opinión de empleadores, de la Universidad Autónoma de Baja California, México: Ed. Miguel Ángel Porrúa; 2006. p. 9-11.

4 Coronado, H.M y Tapia. N.A.. Vinculación universidad-sector productivo. Banco Nacional de Comercio Exterior Vol. 46, No. 10, México, 1996: 2-11 (consultado 8/II/2008) (on line ) Disponible en http://www.ladb.unm.edu.

5 Hernández-Sampieri .R, Fernández Collado C., Baptista-Lucio.P. Metodología de la investigación. $4^{\circ}$ Ed. McGrawHill, México.2006. p.102

6 Sánchez, G. A. G. Los profesionales de ciencias de la salud en el mercado laboral. En Rev. Investigación en Salud. Vol 4, No 001 Abril, Universidad de Guadalajara, México; 2002: 1- 11.

7 Salazar P.T., Trujillo C.C., Gómez M.L. Desempeño profesional de los egresados del programa educativo licenciatura en Enfermería escolarizada. En Eje Temático la Investigación y el Postgrado, soportes para la formación y la vinculación. Facultad de Enfermería Benemérita Universidad Autónoma de Puebla México: 1-10.
8 Salazar P.T; Trujillo C.C.; y Gómez M.L. Op cit. p. 12

9i Sánchez G.A.G, Op cit. p.11

10 Estrella V. G, Ponce L.M. Impacto laboral de egresados universitarios y opinión de empleadores. Universidad Autónoma de Baja California, Colección Problemas Educativos de México, Editor Miguel Ángel Porrúa: México; 2006. p. 215 -24

11 Salazar, op cit : 10

12 Estrella V.G.; Ponce L.M Op Cit., op cit $p$,

13 Valencia H.D., Navarrete H.E., Lopez M.K., Burgos F. Estudio de empleadores y de Egresados. Unidad Regional Centro, Universidad de Sonora. Colección Documentos de Investigación Educativa. México. 2004;15-30

14 Gutiérrez, Op cit. p. 8

15 Gutiérrez, Op cit. p15

16 Valencia H.D.; Navarrete H.E.; Lopez M.K.; Burgos F. Op cit. p- 43

17 Sánchez G.A.G. Op cit.

18 Estrella V.G.; Ponce L.M. Op cit. p.78-120

19 Estrella V.G.; Ponce L.M. Op cit. p. 134-140

20 Salazar P.T; Trujillo C.C.; y Gómez M.L. Op cit. p. 15

\section{CORREO PARA CORRESPONDENCIA}

Mtra. Silvia Crespo Knopler: knopfler36@hotmail.com

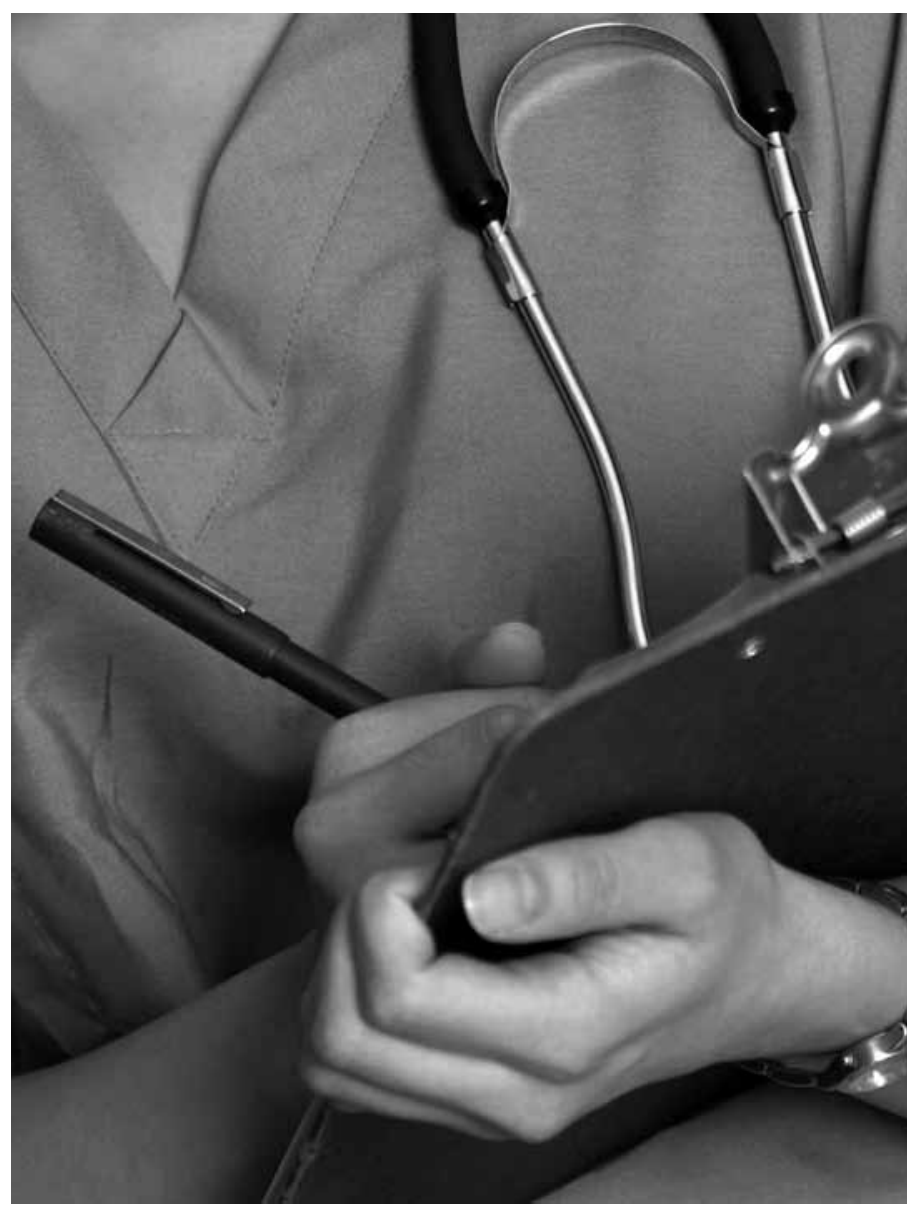

